\title{
Are we at risk of losing the soul of medicine?
}

\author{
Danie Botha, MBChB, DA (SA), MMed (Anesth), MD
}

Received: 5 July 2016/Revised: 17 October 2016/Accepted: 9 November 2016/Published online: 23 November 2016

(c) Canadian Anesthesiologists' Society 2016

Primum non nocere. These three Latin words learned in medical school, "first, do no harm", highlights the physician's primary consideration for the patient's wellbeing. While its author- Hippocrates, Galen, or Thomas Sydenham-may be in dispute, it is undeniable that every medical intervention carries the potential for harm. ${ }^{1}$ This dictum is the cornerstone of the physician's approach to patient care and remains a pillar of modern healthcare.

The Triple Aim $^{2}$ framework, designed to enhance patient experience, improve population health, and reduce costs, was born during the quest for ideal healthcare. The three goals of the framework are laudable, and it is likely that the Triple Aim measures will eventually be achieved. Nevertheless, an additional goal, namely, care of the healthcare provider, ${ }^{2}$ has received inadequate attention. Have we lost sight of the caregiver in our focus on the patient?

Perhaps this oversight relates to the perception of physicians that arose during the "golden age" of medicine (1900-1970). During that era, physicians were heralded as examples of strength and invincibility. More recently, however, physicians' limitations, vulnerability, and even their need for support are being appreciated. Despite this welcome transition, I would contend that physicians strive for perfection and still regard vulnerability as a sign of weakness, which may partly explain why the phenomenon of physician burnout and suicide has been addressed so inadequately. ${ }^{3}$

D. Botha, MBChB, DA (SA), MMed (Anesth), MD ( $₫)$

Department of Anesthesia, Victoria General Hospital, University

of Manitoba, Winnipeg, MB, Canada

e-mail: daniebothawriter@gmail.com
This shortcoming is rather surprising given that burnout and suicide are relatively common occurrences in healthcare staff. For example, more than $50 \%$ of recently surveyed physicians in the United States report burnout, which is considerably higher than the incidence observed in the general population. ${ }^{4,5}$ The irony of physician burnout is that its sufferers often seem to be the most dedicated, conscientious, responsible, and motivated individuals. ${ }^{5}$ With regard to suicide, this tragic end may be considered an occupational hazard of being a doctor. Indeed, compared with the general population, physicians are at a substantially lower risk of dying for all causes of death except suicide. ${ }^{5-8}$ In the United States, 300-400 physicians and medical students lose their lives annually to suicide. ${ }^{9,10}$ Moreover, this may be an underestimation due to miscoding on death certificates because of reluctance to report depression and suicide in this population. ${ }^{7}$

Why the elevated risk of suicide? Suicide is often attributed to untreated or inadequately treated stress, depression, alcoholism, or drug abuse. Nevertheless, one should bear in mind that suicide is a way to die- it is not a root cause of death, ${ }^{5}$ and furthermore, not all suicides are "complications" of depression. Think of the terminally ill patient who opts for physician-assisted suicide or the motivation driving terror-related suicide bombers. ${ }^{5}$ In fact, contemporary suicidology questions the traditional psychiatric approaches that rely heavily on dubious "psychological autopsies", and there is increasing evidence to abandon this practice. ${ }^{11}$

The typical physician suicide victim ${ }^{7,9}$ differs from the non-physician suicide casualty. Doctors are less likely to fail in their attempt because of the lethal combination of the knowledge and accessibility of the means to take one's life. Although physicians are more likely to have elevated barbiturate and benzodiazepine blood levels on routine 
toxicology screening, they are less likely than nonphysicians to be prescribed medication for a diagnosed depression. ${ }^{10}$ They are more likely married (although recent divorce increases the likelihood of suicide) and older, and the suicide is more often attributed to workrelated stress than to a death in the family.

Studies have shown high rates of anxiety and depression among medical students, but relatively few studies have examined the prevalence of mental health issues in physicians. ${ }^{10,12,13}$ For health care workers, depression, burnout, and suicidal ideation have been linked causally to a problematic work environment. Unrealistic demands from patients, increasing lack of autonomy, a chaotic workplace, time pressure, co-worker conflict, and discordance between physician values and those of managers and administrators contribute to an untenable situation. ${ }^{10,12}$

Harassment, bullying, and intimidation are unexplored but serious considerations in the work environment that may have long-term negative effects on one's sense of wellbeing and mental health. Shockingly, victims of abuse (medical students and physicians) may experience indifference and even shaming similar to that of rape victims. $^{12-14}$ The culture of such abuse has been acknowledged for some time, $, 12,13$ and various undergraduate and postgraduate accreditation bodies now routinely screen for this conduct. Nevertheless, it is deplorable that such behaviour still continues with potentially devastating consequences.

Stigmatizing ${ }^{12}$ those who experience depression as being weak, incompetent, or unfit can make it difficult for sufferers to seek the appropriate and timely help they require. It is not uncommon for those who seek help to encounter limited sympathy from colleagues, and surprisingly, they may even face derision, avoidance, and disbelief. The problem may be further aggravated by the tendency of hospitals, licensing boards, and regulatory medical colleges to impose punitive measures on physicians with mental health needs. For example, hospital privileges and professional advancement can be compromised. Annual medical license renewal forms used in Canada and the United States often group mental health questions with inquiries about criminal offences and convictions, implying that these medical issues are uniquely unsavoury. Depression, anxiety, burnout, and suicidal ideation are serious medical conditions that may affect a physician's ability to practice medicine in the same way as poorly controlled coronary artery disease, diabetes, obesity, and sleep apnea. Why do hospitals and licensing bodies differentially approach these latter conditions?

Multiple barriers dissuade physicians from seeking help for serious mental health issues. Such hurdles include reluctance to seek help for any personal illness-with approximately $1 / 3$ of physicians receiving no regular medical care ${ }^{9}$; reluctance to treat fellow physicians; reporting symptoms and/or receiving treatment can trigger repetitive and intrusive examinations, practice restrictions, and increased supervision ${ }^{12}$-all with potentially considerable financial implications; and change of workplace is difficult for those experiencing harassment.

The annual assessment should focus not only on a physician's clinical performance (competence) but also on empathy, optimism, and burnout ${ }^{15,16}$ using well-established tools (e.g., Jefferson Scale of Empathy, ${ }^{17}$ Life Orientation Test-Revised, ${ }^{18}$ Maslach Burnout Inventory).

We must overcome our collective numbness and indifference to mental health issues. When tragedy does strike, a more effective response would be a full timely acknowledgment of the problem with availability of professional counselling and support for the affected staff and colleagues. At the University of California San Diego Medical school, a Healer Education Assessment and Referral (HEAR) program ${ }^{13}$ has been implemented for students, physicians, staff, and administrators. Its goals are to 1) destigmatize those seeking/needing help, 2) identify those at risk (depression and suicide risk), 3) reduce abuse in hospitals and medical schools, 4) refer, without punitive measures, those needing help, and 5) treat and heal traumatized colleagues and students.

An overhaul of annual licensing body questionnaires is needed, particularly with respect to their tone, intention, and function regarding mental health issues. The duty to report mental health issues should be treated as other chronic non-communicable diseases. One may well ask whether the time has come for a "whistleblower-type" program to help identify those in crisis. I acknowledge that this will raise concerns regarding invasion of the privacy and confidentiality of the suffering physician and medicolegal risk for the concerned colleague. There is an established culture of secrecy amongst physicians and administrators, which is likely heightened by a healthcare environment that places a high value on patient confidentiality and privacy. In the end, this may prove to be to the detriment of our colleagues at risk.

There must be a zero tolerance policy for abuse of any kind. This policy needs to be taught and practiced, beginning in medical school and continuing throughout residency and fellowship training programs and beyond. The zero tolerance policy must be embraced as a core institutional value that applies to all and should not be viewed simply as a necessary bureaucratic requirement imposed by accrediting bodies.

Lastly, we must offer better exit strategies to our physicians when they require a time for healing, whether from emotional or other chronic medical conditions. It 
would be helpful to remind and encourage physicians to acquire adequate disability insurance to protect themselves and their family should an unfortunate (and unanticipated) crisis arise. Perhaps we can learn from our co-workers in the nursing field who can move senior members out of the constant stressful work situations into teaching and education positions without marginalization, shaming, or loss of income This should be doable, especially in academic departments of anesthesia, using a pooled remuneration scheme that can easily take advantage of the natural "life cycle" of its members.

In spite of scientific and technological advances, modern medicine is at risk of losing its raison d'être - indeed its soul. Healthcare is at risk of becoming "sick care" with its predominant focus on reactive medicine. Instead, it should once again become our goal to empower caregivers, decision makers, and the public alike to create health-in effect, to prevent and reverse lifestyle diseases and promote wellness. While it remains a truism that "first, do no harm" is the principle that healthcare workers should apply to all decision making, it is important to add that the physician, too, must not be harmed while helping others. The time has come to learn four new Latin words, ultimum, medico nihil nocet, last, do the physician no harm.

The choice is ours.

\section{Sommes-nous en train de perdre l'âme de la médecine?}

Primum non nocere. «Avant tout, ne pas nuire ». Cette locution latine, apprise à l'École de médecine, met en évidence la préoccupation première du médecin : le bien-être de son patient. Bien qu'on ne s'accorde pas toujours sur l'auteur de cette maxime - Hippocrate, Galen ou Thomas Sydenham, il n'en demeure pas moins que chaque intervention médicale porte en elle le potentiel de nuire. ${ }^{1}$ Ce dicton représente la pierre angulaire de l'approche du médecin aux soins aux patients et demeure l'un des piliers des soins de santé modernes.

Le cadre du triple objectif, ${ }^{2}$ conçu pour rehausser l'expérience du patient, améliorer la santé de la population et réduire les coûts, est né de la recherche de soins de santé idéaux. Les trois objectifs de ce cadre sont louables, et les mesures du triple objectif seront probablement atteintes. Toutefois, un objectif supplémentaire, c'est-à-dire les soins voués aux professionnels de la santé, ${ }^{2}$ n'a pas reçu l'attention qu'il méritait. Dans notre vision du patient avant tout, avons-nous perdu de vue la personne qui dispense les soins?
Peut-être cet oubli est-il lié à la perception du médecin qui s'est ancrée pendant «l'âge d'or » de la médecine (1900-1970). Les médecins étaient alors encensés comme des modèles de force et d'invincibilité. Plus récemment, toutefois, nous avons pris conscience des limites, de la vulnérabilité et du besoin de soutien des médecins. Mais, malgré cette transition opportune, j'ai encore le sentiment que les médecins aspirent à la perfection et considèrent toujours la vulnérabilité comme un signe de faiblesse, ce qui pourrait expliquer en partie pourquoi le phénomène de l'épuisement professionnel et du suicide des médecins est encore si maladroitement abordé.

Cette lacune est plutôt surprenante : en effet, l'épuisement professionnel et le suicide sont relativement fréquents dans le milieu des soins de santé. Par exemple, plus de $50 \%$ des médecins récemment interrogés aux États-Unis font état d'épuisement professionnel, une incidence considérablement plus élevée que celle observée dans la population générale. ${ }^{4,5}$ L'ironie de l'épuisement professionnel des médecins est que les personnes touchées semblent bien souvent être les plus dévouées, consciencieuses, responsables et motivées. ${ }^{5}$ En ce qui a trait au suicide, cette fin tragique peut être considérée comme l'un des risques du métier de médecin. En effet, par rapport à la population générale, les médecins courent un risque significativement plus bas de mourir, toutes causes confondues - à l'exception du suicide. ${ }^{5-8}$ Aux États-Unis, 300-400 médecins et étudiants en médecine perdent leur vie chaque année en raison d'un suicide. ${ }^{9,10} \mathrm{Ce}$ chiffre pourrait même être une sous-estimation, en raison d'une erreur volontaire de codage sur les certificats de décès, étant donné la réticence à rapporter la dépression et le suicide dans cette population. $^{7}$

Pourquoi ce risque élevé de suicide? Le suicide est souvent attribué à un stress, une dépression, un alcoolisme ou une toxicomanie non traités ou traités de façon inadaptée. Toutefois, il faut garder à l'esprit que le suicide est une façon de mourir, non une cause première de décès ${ }^{5}$; de plus, les suicides ne sont pas tous des « complications » d'une dépression. Pensez au patient en phase terminale qui choisit l'aide médicale à mourir ou à la motivation des kamikazes commettant des attentats-suicides. ${ }^{5}$ En fait, la suicidologie contemporaine remet en question les approches psychiatriques traditionnelles qui s'appuient fortement sur des « autopsies psychologiques » douteuses, et les données prônant l'abandon de cette pratique s'accumulent. ${ }^{11}$

La victime typique de suicide chez les médecins ${ }^{7,9}$ ne ressemble pas à celle d'un autre milieu. Un médecin a plus de chances de 'réussir' sa tentative de suicide, car il dispose d'une combinaison mortelle : la connaissance et l'accessibilité aux moyens pour s'ôter la vie. Bien qu'il soit plus probable que les médecins affichent des taux sanguins élevés de barbituriques ou de benzodiazépines lors de 
dépistages toxicologiques de routine, la probabilité qu'il leur soit prescrit un traitement médicamenteux en cas de diagnostic de dépression est moins élevée que chez les non-médecins. ${ }^{10}$ Ils sont plus souvent mariés (bien qu'un divorce récent augmente la probabilité d'un suicide) et plus âgés, et le suicide est plus fréquemment attribué à un stress professionnel qu'à un décès dans la famille.

Des études ont montré des niveaux élevés d'anxiété et de dépression parmi les étudiants en médecine; toutefois, seules quelques études ont examiné la prévalence des problèmes de santé mentale parmi les médecins. ${ }^{10,12,13}$ Chez les travailleurs des soins de santé, on a observé un lien causal entre dépression, épuisement professionnel et idéations suicidaires, et environnement de travail problématique. De multiples facteurs contribuent à cette situation intenable : exigences peu réalistes de la part des patients, manque d'autonomie croissant, lieu de travail chaotique, pressions de temps, conflits avec les collègues et discordance entre les valeurs des médecins et celles des gestionnaires et administrateurs. ${ }^{10,12}$

Le harcèlement et l'intimidation sont des enjeux peu étudiés mais graves dans l'environnement professionnel et pourraient avoir des effets négatifs à long terme sur la perception de bien-être et la santé mentale d'un individu. Fait choquant, les victimes d'abus (étudiants en médecine et médecins) pourraient ressentir de l'indifférence, voire de la honte, à l'instar des victimes de viol. ${ }^{12-14}$ Nous sommes conscients depuis un certain temps de l'existence d'une telle culture de l'abus, ${ }^{9,12,13}$ et divers organismes de certification de premier, deuxième et troisième cycles effectuent désormais des dépistages de routine pour identifier ces comportements. Toutefois, il est déplorable qu'un tel comportement perdure, avec ses conséquences potentiellement dévastatrices.

La stigmatisation ${ }^{12}$ des personnes atteintes de dépression comme étant faibles, incompétentes ou inaptes peut mettre un frein à leurs tentatives à trouver une aide adaptée et opportune. Il n'est pas rare que ceux qui demandent de l'aide ne suscitent qu'une sympathie limitée chez leurs collègues et, ce qui est étonnant, ces personnes pourraient même être confrontées à des réactions de dérision, d'évitement et d'incrédulité. Le problème pourrait être aggravé encore par la tendance des hôpitaux, des organismes d'attribution des permis d'exercice et des collèges médicaux de réglementation, à imposer des mesures punitives aux médecins présentant des besoins de santé mentale. Par exemple, il arrive que les droits hospitaliers et l'ascension professionnelle soient compromis. Les formulaires de renouvellement annuel de la licence médicale utilisés au Canada et aux États-Unis regroupent souvent les questions de santé mentale et celles portant sur les infractions criminelles et les condamnations sous le même en-tête, laissant entendre que ces problèmes médicaux sont tout simplement peu reluisants. La dépression, l'anxiété, l'épuisement professionnel et les idéations suicidaires sont des conditions médicales graves qui pourraient affecter la capacité d'un médecin à pratiquer la médecine, de la même façon qu'une coronaropathie, un diabète, une obésité ou une apnée du sommeil mal contrôlés. La question se pose alors : pourquoi les hôpitaux et les organismes de réglementation traitent-ils ces maladies de façon si différente?

De nombreux obstacles dissuadent les médecins de chercher de l'aide lorsqu'ils sont touchés par des problèmes de santé mentale graves. Parmi ces obstacles, citons une réticence généralisée dans la communauté médicale à demander de l'aide, peu importe la maladie personnelle - environ un tiers des médecins ne reçoivent pas de soins médicaux réguliers ${ }^{9}$; une réticence à traiter des collègues médecins; le fait que la déclaration de symptômes et/ou la réception d'un traitement puisse mener à des examens répétitifs et intrusifs, des restrictions de la pratique, et une augmentation de la supervision $^{12}-$ le tout ayant potentiellement un impact financier important; et le fait qu'un changement du milieu de travail soit difficile pour les personnes souffrant de harcèlement.

L'évaluation annuelle devrait examiner non seulement la performance clinique d'un médecin (sa compétence), mais également l'empathie, l'optimisme et l'épuisement professionnel, ${ }^{15,16}$ à l'aide d'outils bien reconnus (par ex. l'Échelle d'empathie clinique de Jefferson, ${ }^{17}$ le Test d'orientation de vie révisée, ${ }^{18}$ ou l'Inventaire d'épuisement professionnel de Maslach).

Il est urgent que nous venions à bout de notre paralysie et de notre indifférence collectives face aux questions de santé mentale. Lorsque survient une tragédie, une réponse plus efficace serait de reconnaître le problème globalement et rapidement, et de mettre à disposition du personnel et des collègues affectés des ressources de counseling professionnel et de soutien. À l'école de médecine de l'Université de Californie à San Diego, un programme intitulé HEAR (pour Healer Education Assessment and Referral - soit Évaluation des connaissances des soignants et consultation) a été mis en œuvre à l'intention des étudiants, des médecins, du personnel et des administrateurs. Les objectifs du programme sont de 1) 'dé-stigmatiser' les personnes qui demandent ou nécessitent de l'aide, 2) identifier les personnes à risque (de dépression ou de suicide), 3) réduire les abus dans les hôpitaux et les écoles de médecine, 4) référer, sans mesures punitives, les personnes ayant besoin d'aide, et 5) traiter et guérir les collègues et étudiants traumatisés.

Un remaniement des questionnaires annuels des organismes de réglementation est nécessaire, tout particulièrement en ce qui touche à leur ton, leur 
intention et leur fonction quant aux questions de santé mentale. Le devoir de rapporter les problèmes de santé mentale devrait être traité comme les autres maladies chroniques non transmissibles. On peut se demander s'il est temps de mettre en place un programme de type «lanceur d'alerte » afin d'identifier les personnes en détresse. Je suis conscient que ce type de programme soulèverait des inquiétudes quant à l'invasion de la vie privée et de la confidentialité du médecin souffrant ainsi qu'un risque médicolégal pour le collègue dénonciateur. Il existe une culture bien établie du secret parmi les médecins et les administrateurs, qui est probablement exacerbée par un environnement des soins de santé qui accorde beaucoup de valeur à la confidentialité et à l'intimité de ses patients. Toutefois, cela pourrait, en fin de compte, être au détriment de nos collègues à risque.

Une politique de tolérance zéro envers tout abus, de quelque nature que ce soit, est essentielle. Cette politique doit être enseignée et exercée, en commençant à l'école de médecine et en continuant tout au long des programmes de formation en résidence et postdoctorale, puis au-delà. Cette politique de tolérance zéro doit être adoptée et promue en tant que valeur institutionnelle fondamentale s'appliquant à tous et ne devrait pas être perçue uniquement comme une simple exigence bureaucratique imposée par les organismes de certification.

Enfin, nous devons offrir de meilleures stratégies de sortie à nos médecins lorsqu'ils ont besoin de temps pour guérir, que ce soit émotionnellement ou en raison d'autres conditions médicales chroniques. Il serait utile de rappeler aux médecins, et de les encourager, à prendre une assurance invalidité adaptée afin de se protéger et de protéger leurs familles si une crise malheureuse (et inattendue) survenait. Peut-être pourrions-nous suivre l'exemple de nos collègues en soins infirmiers : dans leur milieu de travail, il est possible de transférer les membres plus anciens hors des environnements professionnels constamment stressants vers des postes d'enseignement et de formation et ce, sans marginalisation, humiliation ou perte de revenu. Ce devrait être faisable, particulièrement dans les départements universitaires d'anesthésie, en utilisant un système de rémunération groupée qui peut facilement tirer profit du « cycle de vie » naturel de ses membres.

Malgré des progrès scientifiques et technologiques, la médecine moderne court le risque de perdre sa raison d'être - voire son âme. Les soins de santé risquent de devenir des «soins de maladie», avec leur emphase prédominante sur la médecine réactive. Au lieu de cela, notre but devrait être, une fois de plus, d'habiliter tant les personnes prodiguant les soins, les décideurs et le public à créer de la santé - c'est-à-dire, à prévenir et renverser les maladies liées au style de vie et promouvoir le bien-être. Bien que le cliché de «Avant tout, ne pas nuire » demeure le principe qui devrait être appliqué par les professionnels de la santé pour toute prise de décision, il est important d'ajouter qu'on ne doit pas non plus nuire au médecin pendant qu'il s'occupe des autres. Il est temps d'apprendre quatre nouveaux mots latins, ultimum, medico nihil nocet : finalement, ne pas nuire au médecin.

Ce choix nous appartient.

Conflicts of interest None declared.

Editorial responsibility This submission was handled by Dr. Steven Backman, Associate Editor, Canadian Journal of Anesthesia.

Conflit d'intérêt Aucun.

Responsabilité éditoriale Cet article a été traité par Dr Steven Backman, rédacteur adjoint, Journal canadien d'anesthésie.

\section{References}

1. Smith $C M$. Origin and uses of primum non nocere - above all, do no harm! J Clin Pharmacol 2005; 45: 371-7.

2. Bodenheimer T, Sinsky C. From triple to quadruple aim: care of the patient requires care of the provider. Ann Fam Med 2014; 12: 573-6.

3. Legha RK. A History of physician suicide in America. J Med Humanit 2012; 33: 219-44.

4. Shanafelt TD, Hasan $O$, Dyrbye $L N$, et al. Changes in burnout and satisfaction with work-life balance in physicians and the general US working population between 2011 and 2014. Mayo Clin Proc 2015; 90: 1600-13.

5. Aasland OG. Physician suicide-why? Gen Hosp Psychiatry 2013; 35: 1-2.

6. Balch $C M$, Shanafelt $T$. Combating stress and burnout in surgical practice: a review. Adv Surg 2010; 44: 29-47.

7. Torre DM, Wang NY, Meoni LA, Young JH, Klag MJ, Ford DE. Suicide compared to other causes of mortality in physicians. Suicide Life Threat Behav 2005; 35: 146-53.

8. National Mental Health Survey of Doctors and Medical Students - October 2013. Available from URL: http://www.beyondblue. org.au. Available at: www.beyondblue.org.au: http://bit.ly/ 1 VorkGl (accessed October 2016).

9. Andrew LB, Brenner BE. Physician suicide. Medscape Drugs \& Diseases 2015. Available from URL: http://emedicine.medscape. com/article/806779-overview\#showall (accessed October 2016).

10. Gold K, Sen A, Schwenk TL. Details of suicide among US physicians: Data from the National Violent Death Reporting System. Gen Hosp Psychiatry 2013; 35: 45-9.

11. Hjelmeland H, Dieserud $G$, Dyregrov $K$, Dnizek BL, Leenaars $A A$. Psychological autopsy studies as diagnostic tools: are they methologically flawed? Death Stud 2012; 36: 605-26.

12. Shannon $D W$. Fix the root causes of physician burnout. Not just the symptoms. Available from URL: KevinMD.com; http://bit.ly/ 1oqr0sk (accessed October 2016).

13. Downs N, Feng W, Kirby B, et al. Listening to depression and suicide risk in medical students: the Healer Education Assessment and Referral (HEAR) Program. Acad Psychiatry 2014; 38: 547-53 
14. Wible P. Physician Suicide Letters Answered - 2016. Available from URL: www.physiciansuicidelettersanswered.com (accessed October 2016).

15. Center C, Davis M, Detre T, et al. Confronting depression and suicide in physicians - a consensus statement. JAMA 2003; 289: 3161-6.

16. Hojat $M$, Vergare $M$, Isenberg $G$, Cohen $M$, Spandorfer $J$. Underlying construct of empathy, optimism, and burnout in medical students. Int J Med Educ 2015; 6: 12-6.
17. Thomas Jefferson University. Jefferson Scale of Empathy. Available from URL: http://bit.ly/1rpgIvb (accessed October 2016).

18. Scheier MF, Carver CS, Bridges MW. Distinguishing optimism from neuroticism (and trait anxiety, self-mastery, and selfesteem): a reevaluation of the Life Orientation Test. J Pers Soc Psychol 1994; 67: 1063-78. 